\title{
Optimum Design of Organic Electrochemical Type Transistors for Applications in Biochemical Sensing
}

\author{
Shruti Badhwar and K. S. Narayan \\ Chemistry and Physics of Materials Unit, Jawaharlal Nehru Center for Advanced Scientific Research, Bangalore 560064, India
}

Correspondence should be addressed to K. S. Narayan, narayan@jncasr.ac.in

Received 31 May 2008; Accepted 13 August 2008

Recommended by Yasemin Caglar

This paper addresses the issue of optimizing various performance parameters involved in the design of organic electrochemical type transistors based on the conducting polymer, poly (3,4-ethylenedioxythiophene): poly(styrene sulfonate)(PEDOT:PSS) for applications in biochemical sensing. We report the effect of device contact geometry, gate to channel length ratio " $L_{g} / L$," and analyte distance from the source electrode " $x$," on the device sensitivity and response time.

Copyright (c) 2008 S. Badhwar and K. S. Narayan. This is an open access article distributed under the Creative Commons Attribution License, which permits unrestricted use, distribution, and reproduction in any medium, provided the original work is properly cited.

\section{INTRODUCTION}

The discovery of conducting polymers by Shirakawa et al. in 1970s and the subsequent Nobel Prize in Chemistry in 2000 have spurned a huge interest in the field of conducting polymer devices [1]. Electrochemical transistors based on conducting polymers such as poly (3,4-ethylenedioxythiophene): poly(styrene sulfonate) (PEDOT:PSS ) switch between different redox states when a gate voltage is applied through an electrolyte and have shown great promise in the sensing of biochemicals such as glucose [2,3] and DNA [4]. Further, the sensor when operated as a transistor has shown to be more sensitive than a chemiresistor by an order of magnitude [3]. It has also been shown that the gate electrode in an electrochemical transistor can be integrated into the ceiling of a microfluidic channel to realize the "lab-on-chip" concept [5]. However, a major performance constraint of these electrochemical sensors is the response time which ranges from $\sim 60$ seconds to 30 minutes $[3,6]$. This feature is attributed to the large ionic diffusion time scales which are involved in the electrochemical dedoping process. The conducting polymer gets modified from its conducting (oxidized) state to its less conducting (reduced) state, according to the following general electrochemical scheme.
Polymer (Oxidized State) $+\mathrm{M}^{+}+\mathrm{e}^{-} \leftrightarrow$ Polymer (Reduced State), where $\mathrm{M}^{+}$represents cation and $\mathrm{e}^{-}$represents electron [7]. The decrease in conductivity has also been interpreted in terms of an ion-leveraged mechanism, where the disorder driven metal-insulator-transition is characterized by charge localization which is controlled by the ionic environment $[8,9]$. This model interprets the slow response to the percolation of the ions from the electrolyte into the organic film, under the influence of the vertical electric field, affecting the conductance of PEDOT:PSS $[8,9]$.

In this work, we show the effect of additional (parasitic) capacitance, which is prevalent in certain transistor geometries. This factor appears to dominate over the ionic diffusion process and is detrimental to the sensor performance. The performance of these devices is explained in terms of the following parameters: (i) the device sensitivity $(S)$ and (ii) response time $(\tau)$. A phenomenological model built on the basis of the observation indicates an origin to the microscopic structure of the spin-coated PEDOT:PSS blend films. These results can identify and optimize appropriate electrochemical transistor geometry for a given application.

It has been demonstrated in 1980s by Wrigthon et al. that lower source-drain separation or channel length $L$ (50$100 \mathrm{~nm}$ ) leads to higher sensitivity and faster response time 
in gas sensors using solid-state microelectrochemical transistors with conducting polymer Poly(3-methylthiophene) as the active material $[10,11]$. In another device configuration, it has been shown by Berggren et al. that electrochemical transistors designed with (channel width $W /$ channel length $L) \sim 1$ and gate area equal to 10 times WL gives highest on-off ratio and switching speeds [12]. Recent work on modeling of the transistor steady state and transient behavior by Bernards and Malliaras [13] suggests that the gate thickness $H_{g}$, channel length $L$, and the drain voltage $V_{\mathrm{ds}}$ can modify the transient response by the changing the electronic transit time, defined as the $\tau_{e}=L^{2} / \mu V_{\mathrm{ds}}$ and the ionic transit time, defined as $\tau_{i}=C_{d} R_{s} \sim H_{g} / N^{1 / 2},\left(C_{d}\right.$ and $R_{s}$ are the charging capacitance and resistance, $N$ is the ionic concentration) which appear in the following relation:

$$
I_{\mathrm{ds}}(t)=I_{\mathrm{off}}+\Delta I_{\mathrm{ds}}\left(1-f * \frac{\tau_{e}}{\tau_{i}}\right) \exp \left(\frac{-t}{\tau_{i}}\right)
$$

where $I_{\mathrm{ds}}$ is the drain-source current, $I_{\mathrm{off}}$ is the steadystate drain-source current at a gate voltage $V_{\mathrm{gs}}, \Delta I_{\mathrm{ds}}=$ $\left(I_{\mathrm{ds}, V \mathrm{gs}=0}-I_{\mathrm{off}}\right)$ and $f$ are the proportionality constant to account for nonuniformity of the dedoping process. The above model assumes constant carrier mobility and ignores its dependence on electric field and carrier concentration in the polymer. Further, the model attributes the charging capacitance $C_{d}$ to the polarization at PEDOT:PSS-electrolyte interface and the electrolyte-gate electrode interface, but neglects the effect of additional capacitance factors and anisotropic conductivity of PEDOT:PSS in the bulk, interface, and the surface. This simplified first-order model works well with bottom-contact, BC, device geometry (Figure 1(a), inset) in predicting the extent of gating and response times with varying gate properties (size, materials), but may not explain the transistor transfer and transient characteristics with the top-contact geometry, TC (Figure 2). The BC geometry has the drain-source electrodes staggered with respect to the electrolyte/conducting polymer interface, while the TC geometry has the coplanar structure of drain-source/conducting polymer interface and the electrolyte/conducting polymer interface. It may also be mentioned that the organic electrochemical transistor used commonly for biological sensing $[2,5]$ employs TC geometry (Figure 1(b), inset).

In this work, we study the effect of varying (gate length $L_{g}$ /channel length $L$ ), where $L \sim 10 \mathrm{~mm}$, and varying analyte position (distance from source $x$ /channel length $L$ ) on these two different geometries; top contact, TC, and bottom contact, BC. We show that as $L_{g} / L$ is increased; the parasitic capacitance starts to compete with the charging capacitance, observed most prominently in the TC structure. A larger variation of the current density $J(z)$ is expected in TC compared to BC, in the off-state (reduced) of the polymer. The thickness of the polymer and anisotropy in $\sigma$ (bulk \& surface) are factors which decide the magnitude of the offstate conductance. We take these factors into account for interpreting our observations.

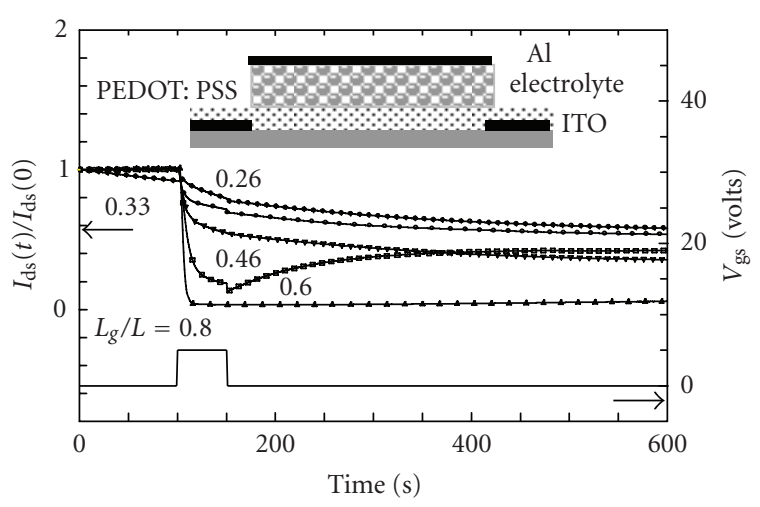

(a)

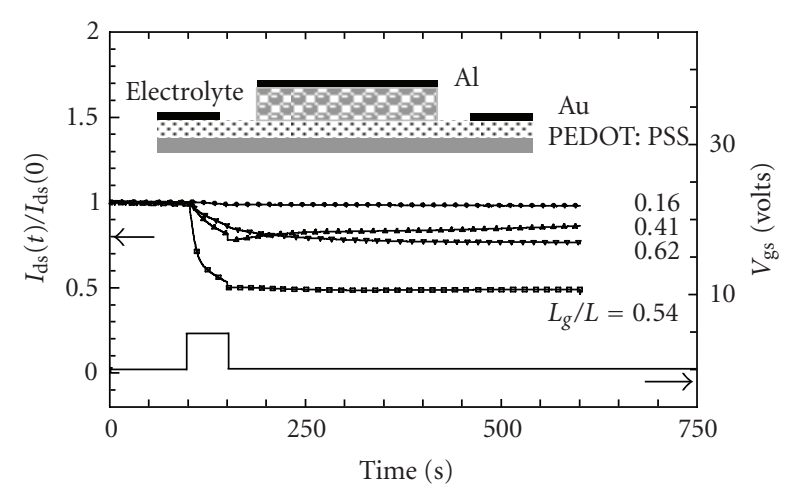

(b)

FIgURE 1: (a) Transient characteristics of electrochemical transistor of $0.1<L_{g} / L<1.0$, with BC geometry, when a $V_{\mathrm{gs}}$ pulse of $5 \mathrm{~V}$, duration 50 seconds, is applied. Inset shows the $\mathrm{BC}$ device geometry, with channel length $L=12 \mathrm{~mm}$, electrolyte thickness $H_{g}=1 \mu \mathrm{m}$, PEDOT:PSS thickness $H_{\text {PEDOT:PSS }}=200 \mathrm{~nm}$, ITO thickness $H_{\text {ITO }}=150 \mathrm{~nm}$, and $L_{\text {elec }}=L_{g}$. (b) Transient characteristics of electrochemical transistor of $0.1<L_{g} / L<1.0$, with TC geometry, when a $V_{\mathrm{gs}}$ pulse of $5 \mathrm{~V}$ duration 50 seconds, is applied. Inset shows the TC device geometry, with channel length $L=12 \mathrm{~mm}$, electrolyte thickness $H_{g}=1 \mu \mathrm{m}$, PEDOT:PSS thickness $H_{\text {PEDOT:PSS }}=150 \mathrm{~nm}$, Au thickness $H_{\mathrm{Au}}=70 \mathrm{~nm}$, and electrolyte length $L_{\text {elec }}=L_{g}$.

\section{EXPERIMENTAL METHODS}

The $\mathrm{BC}$ and $\mathrm{TC}$ device structures are shown in Figures 1 and 2 , inset. A set of 6-8 electrochemical transistors with $L \sim 12 \mathrm{~mm}$ and width $W \sim 3 \mathrm{~mm}$ was fabricated. Commercially available Baytron (P) PEDOT:PSS was spin-coated on precleaned glass substrates following standard procedures. PVA ( molecular weight $\sim 130000)$ and $\mathrm{KCl}(250 \mathrm{mM})$ in the ratio (1:9), baked at $90^{\circ}$ for 4 hours, were used as the solidelectrolyte. Further fabrication details for these solid-state devices are mentioned in $[8,14]$. In all our devices, the length of the electrolyte, $L_{\mathrm{elec}}$, is equal to the length of gate electrode, $L_{g}$. To study the sensor performance on the position of the liquid analyte, (Figure 5, inset), a $\sim 70 \mathrm{nl}$ drop of $250 \mathrm{mM}$ $\mathrm{KCl}$ solution was positioned on the PEDOT:PSS channel at varying $x$ using Auto Nanojet injector and micromanipulator from Warner instruments. The gate voltage was applied through the electrolyte using a gold-coated probe tip. All 


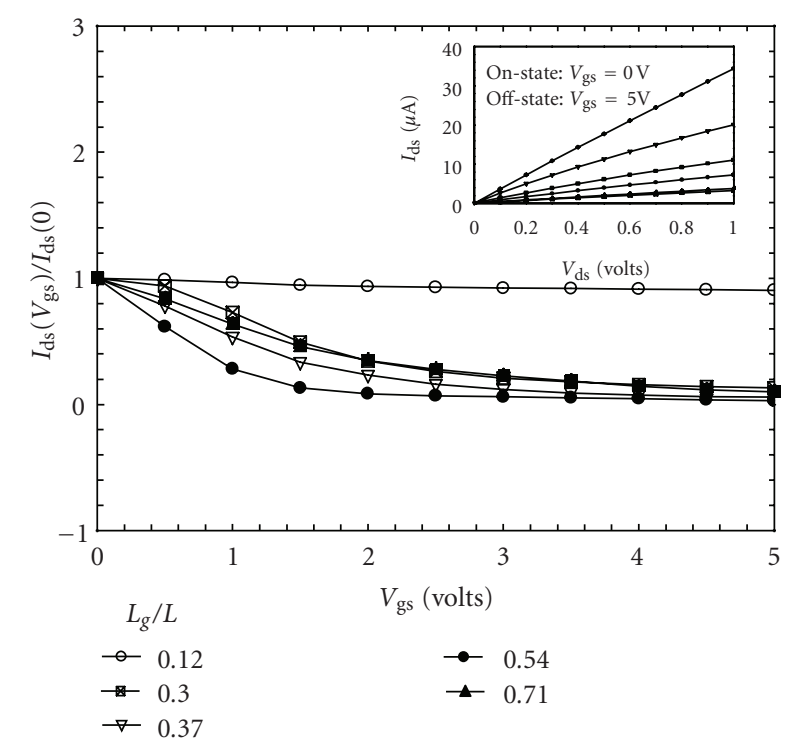

FIgure 2: Transconductance characteristics of electrochemical transistor of $0.1<L_{g} / L<1.0$, with TC geometry. Inset shows the steady state, $I_{\mathrm{ds}}$ versus $V_{\mathrm{ds}}$ characteristic of an electrochemical transistor at varying $V_{\mathrm{gs}}$. The transistor is normally "on" at $V_{\mathrm{gs}}=$ $0 \mathrm{~V}$.

electrical measurements were carried out using Kiethley Sourcemeter, Model 2400 and Electrometer, Model 6514, and controlled by Lab view interface software.

\section{RESULTS AND DISCUSSION}

Transistor characteristics were obtained on a set of devices with similar initial drain-source conductance values and different $L_{g} / L$ ratios. The $I_{\mathrm{ds}}-V_{\mathrm{ds}}$ response, transfer, or curve $\left(I_{\mathrm{ds}}\right.$ versus $\left.V_{\mathrm{gs}}\right)$ and transient behavior $I_{\mathrm{ds}}(t)$ were determined for each of the device. With $V_{\mathrm{ds}}$ swept from 0 to positive $1 \mathrm{~V}$ and $V_{\mathrm{gs}}$ varied in discrete steps from 0 to $+5 \mathrm{~V}$, the PEDOT:PSS channel was observed to switch from its normally on to the off state (see Figure 2, inset). $I_{\mathrm{gs}}$ magnitude was negligible compared to $I_{\mathrm{ds}}$. The transfer or transconductance characteristics were obtained by keeping $V_{\mathrm{ds}}$ constant at positive $1 \mathrm{~V}$ and by sweeping the gate voltage cyclically from 0 to $+5 \mathrm{~V}$ and then to $-5 \mathrm{~V}$. The hysteresis in the transfer characteristics can be attributed to asymmetric charging and discharging rates of the electrolyte capacitance. The hysteresis and the slope of transfer curve (i.e., the transconductance $G_{m}$ ) were observed to be a function of the $L_{g} / L$, with $G_{m}$ increasing monotonically with $L_{g} / L(0.1$ to 1.0$)$ for BC geometry with a maxima at $0.2<L_{g} / L<0.5$ for the TC geometry. Figure 2 shows the transconductance characteristics when $V_{\mathrm{gs}}$ was swept from 0 to $+5 \mathrm{~V}$ for a device with TC geometry.

The transient characteristics (Figure 1) were obtained by applying a gate voltage pulse $V_{\mathrm{gs}}=5 \mathrm{~V}$, for a duration of 100 seconds to the gate electrode, at a constant drain voltage, $V_{\mathrm{ds}}=1 \mathrm{~V}$. The change in the $I_{\mathrm{ds}}$ from on to the off state is depicted as $\Delta I_{\mathrm{ds}}$. The current drop is normalized with the initial $I_{\mathrm{ds}}\left(V_{\mathrm{gs}}=0 \mathrm{~V}\right)$ and defined as $\beta=\Delta I_{\mathrm{ds}} / I_{\mathrm{ds}}$. A scaling parameter for the sensitivity $S$ defined as the ratio of $\beta$ to $L_{g} / L$, that is, $S=\beta /\left(L_{g} / L\right)$ is useful in characterizing the device response to the electrolyte and device dimensions. The time constant $\tau$ (see Figure 4 ) can be extracted by fitting (1) to the experimentally observed transient characteristics.

Figure 4 represents $\tau$ and $S$ (indicated by the color scale) dependence on the $L_{g} / L$ ratios. In the BC device geometry, $\tau$ decreases by more than an order of magnitude (60 seconds to 0.5 second), with increase in $L_{g} / L$ from 0.1 to 1.0 . The decrease in $\tau$ can be better expressed in terms of the slope $\Delta \tau / \Delta\left(L_{g} / L\right) \sim 66$. The sensitivity, depicted in blue in Figure 4 , is almost constant at $S \sim 1$ for the entire range of $L_{g} / L$. The operating window of the BC device for optimum performance (high $S$ and low $\tau$ ) is then observed to be $0.9<$ $L_{g} / L<1.0$. For the entire range of the $L_{g} / L$, the $S$ of TC $>S$ of BC. In case of TC device geometry, high sensitivity $(S \sim 2.5)$ is achieved in the range $0.2<L_{g} / L<0.5$, with a corresponding $\tau$ value of 6 seconds. It is to be noted that this suggested operating window for $\mathrm{BC}$ and TC geometries was obtained statistically by repeating the measurements over a set of 8 batches $\left(1\right.$ batch $=6$ devices with $\left.0.1<L_{g} / L<1.0\right)$.

The response function of $\tau\left(L_{g} / L\right)$ and the higher magnitude of $\tau$ in the TC geometry as compared to BC geometry seem to suggest a competing factor from the additional parasitic capacitance in determining the device performance. The parasitic capacitance in TC geometry (Figure 2) essentially comprises of two components: (i) outer fringing capacitance, $C_{\mathrm{fgs}}$ and $C_{\mathrm{fgd}}$ (between outer sides of the gate to source/drain electrode across the air-dielectric) and (ii) outer fringing capacitance $C_{\mathrm{fgsp}}$ and $C_{\mathrm{fgdp}}$ (between outer sides of the gate electrode to the PEDOT:PSS film on either side of the electrolyte, across the air-dielectric). To account for the parasitic capacitance in the electrochemical transistors, (1) can be modified to $I_{\mathrm{ds}}(t)=I_{\mathrm{off}}+\Delta I_{\mathrm{ds}} \exp \left(-t / R C_{\text {eff }}\right)$, for $f=0$, where $\tau=R C_{\text {eff }}$ is the effective response time. The response time $\tau$ for TC configuration is a nonlinear function and can be represented by an expression consisting of contributions from the intrinsic factors, $f\left(L_{g} / L\right)$, and the extrinsic parasitic factors, $g\left(L_{g} / L\right)$.

These results can also be understood from microscopic aspects of the conducting polymer film organization. It has been reported [14] that the bulk conductivity in spin-coated PEDOT:PSS thin films can be three orders in magnitude less than the lateral conductivity in the plane of the film. This is because in the perpendicular direction, the PEDOTrich domains are separated by thick barriers formed by PSS lamella, whereas in the parallel direction, within the PEDOTrich lamella, conduction can take place with a thin or no barrier at all by 3D variable-range hopping. The current (when the polymer is in the reduced nonconducting form at the interface) through the lower bulk conductivity is lowered more effectively. In the off state of the TC device, the leakage pathways are clearly reduced compared to the BC device and can explain the higher $S$ values. The effect of parasitic capacitance and the anisotropic PEDOT:PSS conductivity on the performance parameters of the electrochemical transistor can be better understood in terms of an equivalent (lumped) circuit model, is shown in Figure 3. The lateral resistance, $R_{\text {bulk }}<$ the vertical resistance, $R_{\text {ver }}$. The interface resistance 


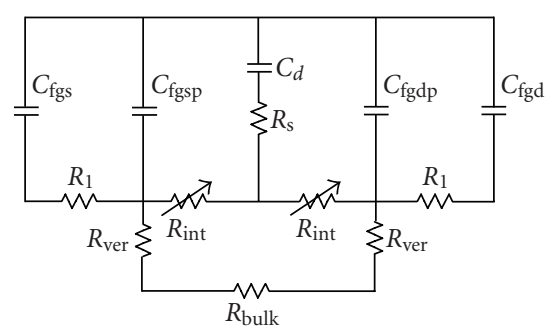

Figure 3: Equivalent circuit representation of an electrochemical transistor, including the effect of additional (parasitic) capacitance and the nonuniform conductivity of PEDOT:PSS.

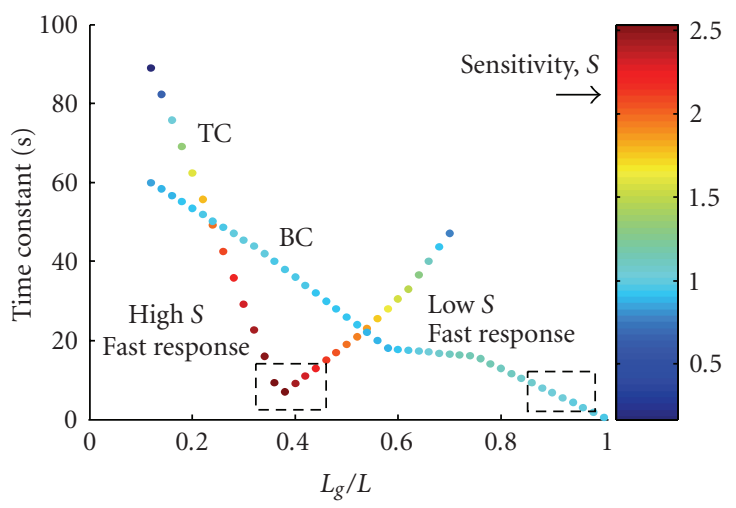

FIgURE 4: Response time, $\tau$, shown on the $y$ axis and sensitivity, $S$, shown as the color bar versus $L_{g} / L$ of the electrochemical transistor with $\mathrm{BC}$ and $\mathrm{TC}$ device geometry.

$R_{\mathrm{int}}$ is represented as a variable resistor. The resistance of the PEDOT:PSS film at the surface, on either side of the electrolyte, is denoted as $R_{1}$. The fringing capacitances, defined previously, are accounted in the circuit diagram.

The charging capacitance $C_{d}$ and resistance $R_{s}$ for electrochemical transistors in $\mathrm{BC}$ and TC geometry, $0.1<L_{g} / L<$ 1.0 , were measured using the 4200-CVU (multifrequency impedance card) inserted in Kiethley parameter analyzer, in a series $\mathrm{RC}$ configuration. The transistors were driven at $10 \mathrm{KHz}, 30 \mathrm{mV}$ rms $V_{\mathrm{gs}}$ signal riding over a DC bias of $-5.0 \mathrm{~V}<V_{\mathrm{gs}}<5.0 \mathrm{~V}$, with drain-source electrodes shorted (figure not included). Unlike the conventional silicon-based MOSFET, where CV measurements are made to extract useful device parameters such as oxide capacitance, flat band capacitance, and oxide thickness, the inference from $\mathrm{CV}$ measurements of electrochemical transistors is more involved and must be drawn with care. This is because in an electrochemical transistor, an array of charged species and oriented dipoles exists at the metal-electrolyte interface and forms an electrical double layer [15]. At a given potential $V_{\mathrm{gg}}$, the metal-electrolyte interface is characterized by a double layer capacitance $C_{d}$. However, unlike real capacitors, whose capacitances are independent of the voltage across them, $C_{d}$ is often a function of potential, and hence the device geometry which is an important criteria in the electric field distribution. Furthermore, the exact distribution profile of

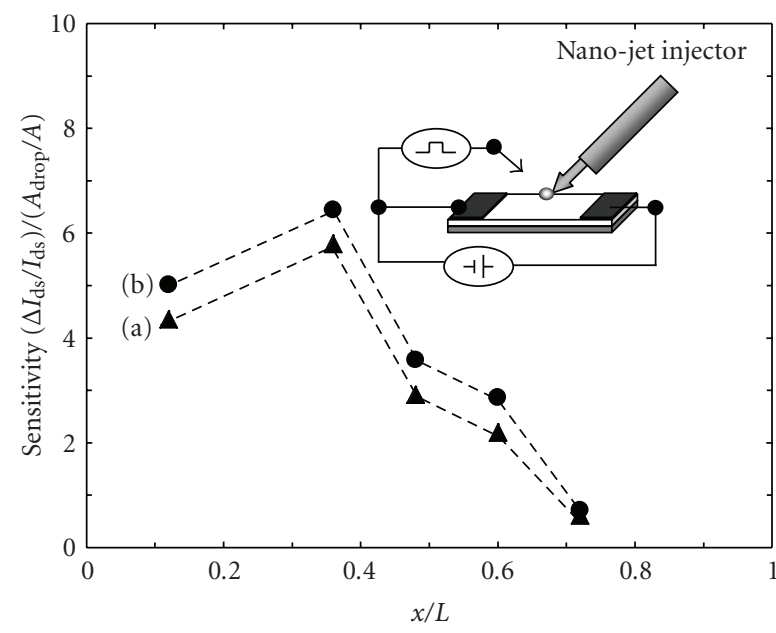

FIGURE 5: Sensitivity, $S$ versus $x / L$ for an electrochemical transistor, TC Geometry, plotted for different positions of the analyte (a) at $V_{\mathrm{gs}}=0 \mathrm{~V}(\mathrm{~b})$ at $V_{\mathrm{gs}}=0.2 \mathrm{~V}$, duration 100 seconds. The inset shows the drop-potentiometry setup.

the ions in the bulk, their transport, and influence on the potential must be known, to obtain a suitable value of $C_{d}$.

From our measurements, the general trend that $C_{d}$ (when $V_{\mathrm{gs}} \sim-3.0 \mathrm{~V}$, PEDOT:PSS is in oxidized state, and the transistor is on) $>C_{d}$ (when $V_{\mathrm{gs}} \sim+5.0 \mathrm{~V}$, PEDOT:PSS is in reduced state, and the transistor is off) is observed. The magnitudes of $C_{i}=\left(\int C_{d} d V_{\mathrm{gs}}\right) /\left(\int d V_{\mathrm{gs}}\right)$, where $C_{i}$ is the integral capacitance averaged over changes in potential, are consistent with that evaluated from geometric parameters and reasonably assumed value of dielectric constant $(C=$ $A \varepsilon / t \sim 0.01$ to $0.15 \mathrm{nF}$ ). The scaling trend in CV measurement is also qualitatively in agreement with the $S$ and $\tau$ measurements. The specifics involving measurement of individual components of capacitance and resistance, their relationship to $S\left(L_{g} / L\right)$ and $\tau\left(L_{g} / L\right)$ are quite complex owing to factors like geometrical imprecision, film morphology, humidity, irreversibility and so forth and require separate effort. An accurate electrostatic modeling which is underway in our laboratory can possibly arrive at a form of $S\left(L_{g} / L\right)$ and $\tau\left(L_{g} / L\right)$, where the minima correspond to the experimentally observed optimum value of $\left(L_{g} / L\right)$.

These observations, results, and interpretation are also consistent with drop-potentiometry type measurements, where the electrolyte is in form of a liquid drop with a thin wire electrode immersed in it. The transient response of the electrochemical transistor to a liquid electrolyte gated, with a pulse of $V_{\mathrm{gs}}=0.2 \mathrm{~V}$, duration 100 seconds, and constant $V_{\mathrm{ds}}=1 \mathrm{~V}$, was studied. The drop was precisely positioned at distance $x$ from the source electrode, for 6 devices with $0.1<$ $x / L<1.0$. The rate of droplet evaporation, being a constant for each of the " $x$ " positions, did not affect the relative measurements. $V_{\mathrm{gs}}$ of sufficiently low magnitude was used to prevent hydrolysis. The sensitivity, defined as $S=\beta /$ (area of drop/channel area), is observed to follow similar qualitative trend (Figures 4 and 5), with $S$ attaining a maxima at $x / L \sim 0.4$ for an electrochemical transistor with a TC geometry. These 
results also indicate the requirement of appropriate location for dispensing the drop to yield high performance.

\section{SUMMARY}

We report observations which indicate the requirement for optimizing the geometrical factors in designing polymerbased electrochemical transistors for sensor applications. These results were analyzed in terms of the switching response and a sensitivity parameter represented by a scaleddimensional factor. The results were qualitatively interpreted in terms of additional parasitic capacitance factors and the anisotropy in the conductivity of the conductivity polymer medium.

\section{ACKNOWLEDGMENTS}

The authors would like to acknowledge the Department of Science and Technology for supporting the work. They also acknowledge V. Gautam for partial assistance in measurement.

\section{REFERENCES}

[1] H. Shirakawa, E. J. Louis, A. G. MacDiarmid, C. K. Chiang, and A. J. Heeger, "Synthesis of electrically conducting organic polymers: halogen derivatives of polyacetylene, $(\mathrm{CH})_{x}$," Journal of the Chemical Society, Chemical Communications, no. 16, pp. 578-580, 1977.

[2] Z.-T. Zhu, J. T. Mabeck, C. Zhu, N. C. Cady, C. A. Batt, and G. G. Malliaras, "A simple poly(3,4-ethylene dioxythiophene)/poly(styrene sulfonic acid) transistor for glucose sensing at neutral pH," Chemical Communications, vol. 10, no. 13, pp. 1556-1557, 2004.

[3] D. J. Macaya, M. Nikolou, S. Takamatsu, J. T. Mabeck, R. M. Owens, and G. G. Malliaras, "Simple glucose sensors with micromolar sensitivity based on organic electrochemical transistors," Sensors and Actuators B, vol. 123, no. 1, pp. 374378, 2007.

[4] K. Krishnamoorthy, R. S. Gokhale, A. Q. Contractor, and A. Kumar, "Novel label-free DNA sensors based on poly $(3,4-$ ethylenedioxythiophene)," Chemical Communications, vol. 10, no. 7, pp. 820-821, 2004.

[5] J. T. Mabeck, J. A. DeFranco, D. A. Bernards, G. G. Malliaras, S. Hocdé, and C. J. Chase, "Microfluidic gating of an organic electrochemical transistor," Applied Physics Letters, vol. 87, no. 1, Article ID 013503, 3 pages, 2005.

[6] P. N. Bartlett, "Measurement of low glucose concentrations using a microelectrochemical enzyme transistor," The Analyst, vol. 123 , no. 2, pp. 387-392, 1998.

[7] D. Nilsson, M. Chen, T. Kugler, T. Remonen, M. Armgarth, and M. Berggren, "Bi-stable and dynamic current modulation in electrochemical organic transistors," Advanced Materials, vol. 14, no. 1, pp. 51-54, 2002.

[8] A. J. Epstein, F.-C. Hsu, N.-R. Chiou, and V. N. Prigodin, "Electric-field induced ion-leveraged metal-insulator transition in conducting polymer-based field effect devices," Current Applied Physics, vol. 2, no. 4, pp. 339-343, 2002.

[9] F.-C. Hsu, V. N. Prigodin, and A. J. Epstein, "Electric-fieldcontrolled conductance of "metallic" polymers in a transistor structure," Physical Review B, vol. 74, no. 23, Article ID 235219, 12 pages, 2006.
[10] S. Chao and M. S. Wrighton, "Solid-state microelectrochemistry: electrical characteristics of a solid-state microelectrochemical transistor based on poly(3-methylthiophene)," Journal of the American Chemical Society, vol. 109, no. 7, pp. 2197-2199, 1987.

[11] E. T. T. Jones, O. M. Chyan, and M. S. Wrighton, "Preparation and characterization of molecule-based transistors with a 50$\mathrm{nm}$ source-drain separation with use of shadow deposition techniques: toward faster, more sensitive molecule-based devices," Journal of the American Chemical Society, vol. 109, no. 18 , pp. 5526-5528, 1987.

[12] M. Bergrren, R. Forchheimer, J. Bobacka, et al., "PEDOT:PSSbased electrochemical transistors for ion-to-electron transduction and sensor signal amplification," in Organic Semiconductors in Sensor Applications, vol. 107 of Springer Series in Materials Science, pp. 263-280, Springer, Berlin, Germany, 2008.

[13] D. A. Bernards and G. G. Malliaras, "Steady-state and transient behavior of organic electrochemical transistors," Advanced Functional Materials, vol. 17, no. 17, pp. 3538-3544, 2007.

[14] J. Lu, N. J. Pinto, and A. G. MacDiarmid, "Apparent dependence of conductivity of a conducting polymer on an electric field in a field effect transistor configuration," Journal of Applied Physics, vol. 92, no. 10, pp. 6033-6038, 2002.

[15] A. J. Bard and L. R. Faulkner, Electrochemical Methods, Fundamentals and Applications, John Wiley \& Sons, New York, NY, USA, 2nd edition, 2000. 

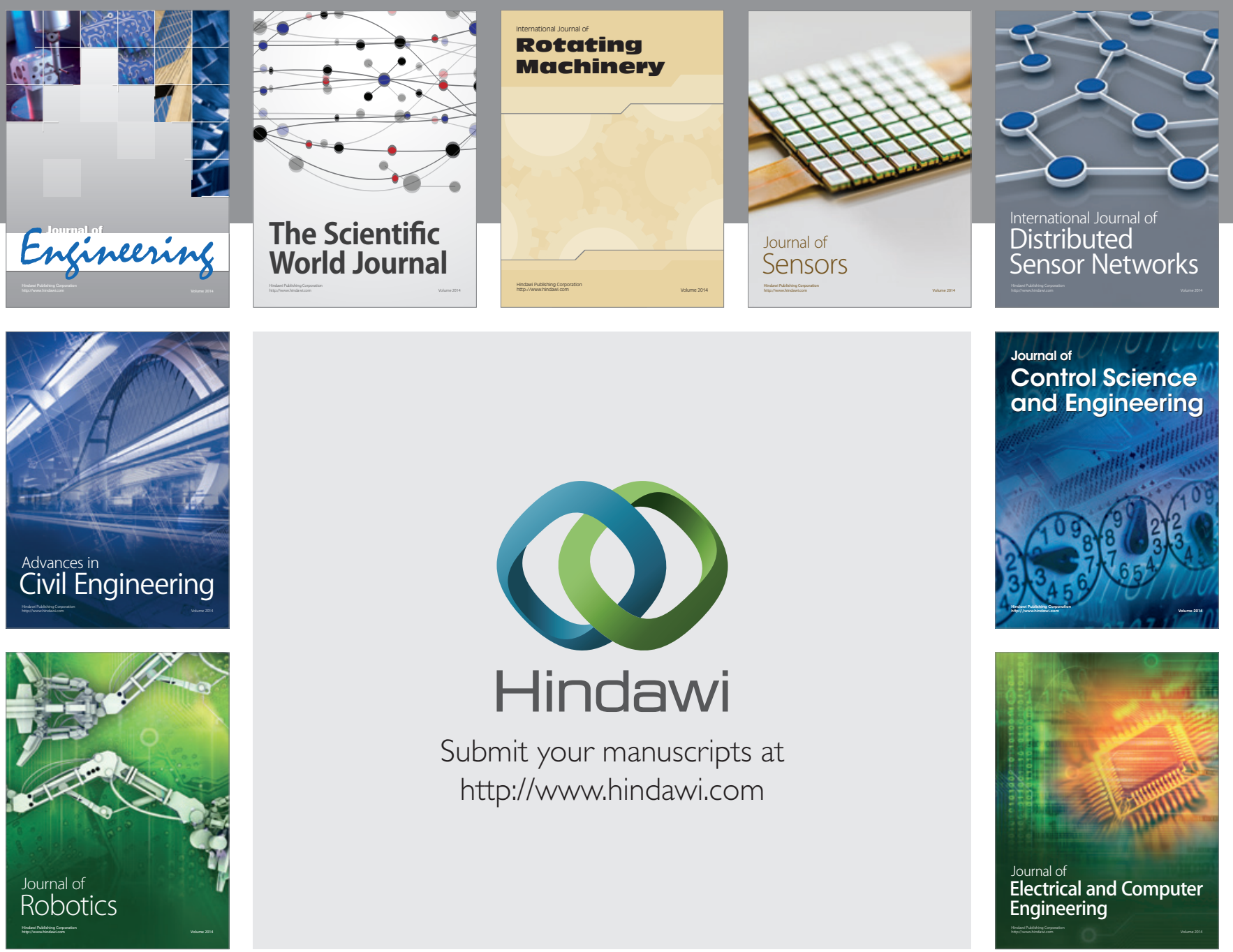

Submit your manuscripts at

http://www.hindawi.com
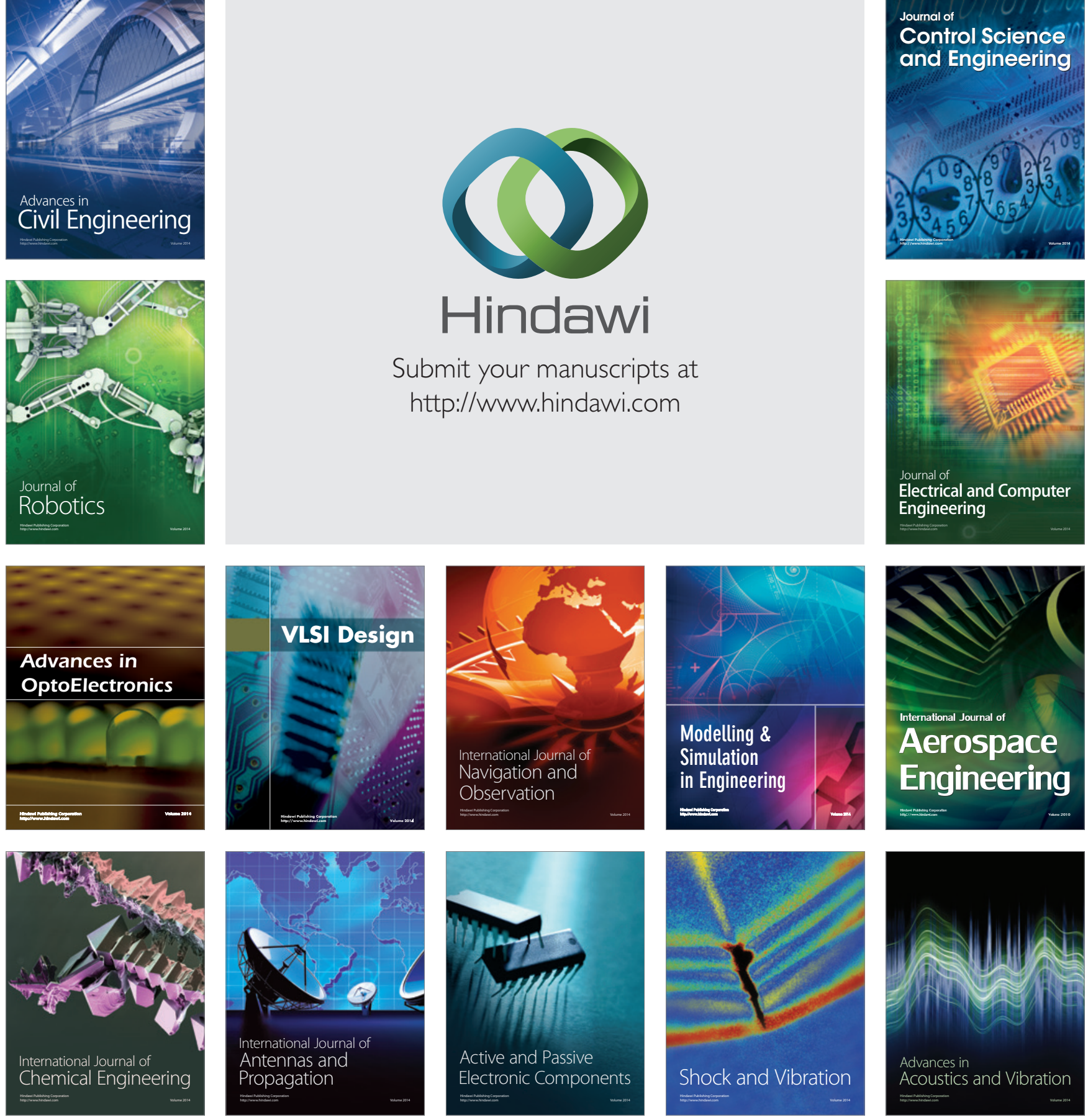